\title{
Root Resorption Incidental to En-masse Retraction in Maxillary Protrusion Cases: A Systematic Review
}

\author{
Heba M. Dehis ${ }^{\# 1}$, Fouad A. El-Sharaby ${ }^{\# 2}$, Yehya A. Mostafa ${ }^{\# 3}$, Faten H. Eid ${ }^{\# 4}$
}

\#1 BDS, MSc, M. Orth RCSEd (UK). Assistant lecturer, Department of Orthodontics and Dentofacial Orthopedics. Faculty of Dentistry, Cairo University. Cairo, Egypt.Phone no: (02)-01020490611, E-mail: drhebadehis@gmail.com

\#2 BDS, MSc, PhD, FDSRCSEd (UK). Assistant Professor, Department of Orthodontics and Dentofacial Orthopedics. Faculty of Dentistry, Cairo University. Cairo, Egypt.Phone no: (02)-01223421238. E-mail: fsharaby@hotmail.com

\#3 BDS, MSc, PhD, FDSRCSEd (UK).Professor and Head of Department of Orthodontics and Dentofacial Orthopedics. Faculty of Dentistry, Future University. Cairo, Egypt.Phone no: (02)01222131934, E-mail: yehya3d@ gmail.com

\#4 BDS, MSc, PhD. Professor of Orthodontics and Dentofacial Orthopedics. Faculty of Dentistry, Cairo University. Cairo, Egypt. Phone no: (02)-01227446428, E-mail: drfaten.eid@ hotmail.com

\section{Abstract:}

The aim of this systematic review was to evaluate the available literature and assess the incidence and degree of external apical root resorption associated with en-masse retraction in maxillary protrusion cases. PubMed, OvidSP,LILACS and Cochrane Central Register of Controlled Trials(CENTRAL), Google Scholar and OpenGrey databases were searchedtill November 2017 to identify all articles relevant to this review. Hand search of orthodontic journals and references list of relevant articles was done during the same time frame. The eligibility criteria included randomized and quazi-randomized clinical trials (RCTs and q RCTs), controlled clinical trials, non-randomized clinical trials and clinical trials. Studies having only non-growing subjects with either Class I or II molar relationship having maxillary protrusion requiring extraction of first premolars were included. Methodological weaknesses were highlighted and the quality of the studies was ranked using MINORS (Methodological Index For Non-Randomized Studies) tool. Search resulted in 1035 citations from which only two articles were eligible tobe included in the qualitative analysis. The included studies were a non-randomizedcontrolled trial and a clinical trial which were given a score of 17 and 14 respectively on the MINORS scoring system where both were below the global ideal score.Low quality evidence denotes that incisors' root resorption associated with en-masse retraction was greatest for maxillary lateral incisors but was clinically insignificant. High quality RCTs evaluating the root resorption associated with en-masse retraction and the average amount of anterior teeth retraction as well as pain associated should be conducted.

Keywords:En-Masse retraction, Root resorption, Maxillary protrusion, Systematic review.

\section{Corresponding author:Heba M. Dehis}




\section{INTRODUCTION:}

External apical root resorption is an unpredictable common adverse effect of orthodontic treatment(1). Many factors have been proposed as potential risk factors. General factors include genetic causes(2), individual susceptibility $(3,4)$ as well as age $(5,6)$. Local risk factors were more correlated with individual variation in root shape such as blunt, pipette or dilacerated roots, previous history of traumatized teeth as well as impacted teeth $(5,6)$. Many predisposing factors were related to the orthodontic treatment as well, including the type and extent of tooth movement $(7,8)$, the amount of force (9)applied together with the mechanics used(10), as well as the duration of treatment(11).

Orthodontic treatment of patients having maxillary protrusion usually implies extraction first premolars and retracting anterior teeth. One technique of anterior teeth retraction that has gained popularity because of its simplicity is the en-masse retraction(12). Like any other technique it has its pros and cons. One of its benefits could bethe assumption that treatment duration is reduced because all anterior teeth are retracted at the same time as one unit instead of retracting the canines first followed by incisors. Thus, many side effects resulting from long treatment duration might be ruled out.

Additionally, better patients' satisfaction could be achieved as extraction space is less vivid using this technique and patients can visualize the improvement on their soft tissue profiles throughout the retraction process without having to wait to the end (13).

On the other hand, the larger scope of teeth retraction being experienced using this technique could be expected to tax the posterior teeth(12), or in case of applying skeletal anchorage modalities greater tooth movement would be expected increasing the risks of root resorption. From another perspective, the mechanics used during retractiondetermines the location of pressure areas within anterior teeth relative to labial or palatal cortical plates of bone which in turn might increase the risks of incidence of this adverse effect(14).

A recentsystematic review(15)evaluated the anchorage during en-masse retraction with different anchorage modalities. Meanwhile, the incidence of root resorption with en-masse retraction techniques and its severity was still a question that needed an answer. Thus, this systematic review aimed to systematically analyze the available literature and evaluate the amount of root resorption associated with en-masse retraction techniques in adults having maxillary protrusion. The secondary outcomes were to assess the average amount of anterior teeth retraction using this technique as well as to evaluate the pain associated during retraction.

\section{MATERIALS AND METHODS:}

The "PIOS" of the current review was set so that the population (P) comprised non-growing patients having maxillary protrusion. The intervention (I) was restricted to extraction of first premolars followed by en-masse retraction of anterior teeth. The primary outcome (O) to be assessed was the incidence and amount of external apical root resorption associated with en-masse retraction in anterior and/or posterior teeth. The secondary outcomes included assessing the mean amount of anterior teeth retraction associated with this technique as well as pain evaluation during retraction. As for the study (S) designs, randomized, quasi-randomized trials, controlled clinical trials, clinical trials and non-randomized controlled/clinical trials were included (Table 1). 


\begin{tabular}{|c|c|c|}
\hline Category & Inclusion criteria & Exclusion criteria \\
\hline $\begin{array}{l}\text { Types of } \\
\text { participants } \\
\text { (P) }\end{array}$ & 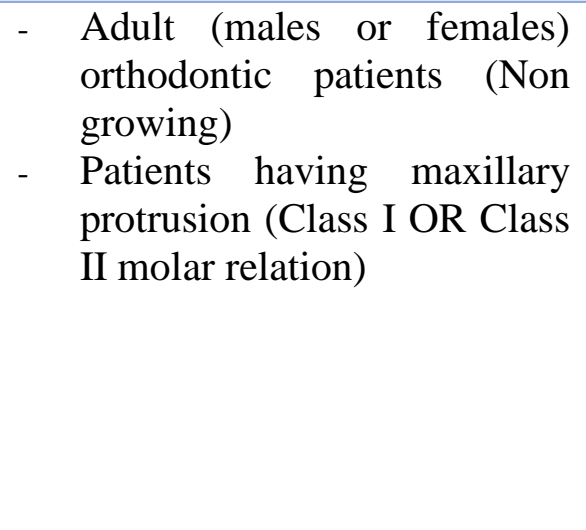 & $\begin{array}{l}\text { - Patients with habits (Thumb sucking, } \\
\text { tongue thrusting, nail biting ...etc) } \\
\text { - Patients with previous trauma/ signs of } \\
\text { root shortening at the start of treatment/ } \\
\text { impaction/ dilacerated teeth. } \\
\text { - Patients suffering from systemic } \\
\text { diseases/ bronchial Asthma/ syndromes/ } \\
\text { dental, pulp, or periodontal problems/ } \\
\text { previously endodontically treated teeth. } \\
\text { - Patients with previous orthodontic } \\
\text { treatment }\end{array}$ \\
\hline $\begin{array}{l}\text { Intervention } \\
\text { (I) }\end{array}$ & $\begin{array}{l}\text { - Treatment by extraction of } \\
1^{\text {st }} \text { premolars } \\
\text { - Using Metal Brackets, slot } \\
0.022^{\prime \prime} \times 0.028 \text { ROTH, or } \\
\text { MBT prescription } \\
\text { - En-masse retraction: } \\
\text { (retraction of canines + } \\
\text { incisors as one unit) }\end{array}$ & $\begin{array}{l}\text { - Treatment by extracting } 2^{\text {nd }} \text { premolars, } \\
\text { molars, or asymmetric extraction } \\
\text { - Using Bi-Dimensional bracket system, } \\
\text { Ceramic brackets, Ceramic brackets with } \\
\text { metal slot, Lingual brackets, Aligners } \\
\text { - Studies employing surgical, } \\
\text { pharmacological or physical methods for } \\
\text { altering the rate of tooth movement or } \\
\text { reducing pain. }\end{array}$ \\
\hline
\end{tabular}

\section{Outcome 1ry outcome:}

(O)
Studies included should report the amount of root resorption of maxillary \&/or mandibular teeth (anterior \&/ or posterior teeth)

2ry outcomes:

- Amount of anterior teeth retraction

- Pain associated with retraction

\section{Study}

design (S)
- Retrospective studies

- Clinical trials with poor methodology

- Experimental (Animal/ in-vitro) studies

- Dental model analysis/Finite Element Analysis studies

- Narrative review

- Literature reviews

- Books/E-Books

- Author's reply/ Letter to editor/ Opinion articles

- Case reports/Case series

- Pilot/ Preliminary studies

- Abstracts 
Electronic search through different databases was performed by the first two authors (H.D and F.S) independently till November 2017 without date restriction,however studies with only English and French languages were included (Table 2). The databases included Medline (via PubMed), Medline (viaOvidSP),LILACS and Cochrane Central Register of Controlled Trials(CENTRAL). Further electronic search for grey literature was done through Google Scholar and OpenGrey databases. Trial registrations, dissertations and conference abstracts were investigated by further search through ClinicalTrials.Gov, ProQuest and Conference Proceedings Citation Index respectively.

Table 2: Databases of published trials.

Database

Search engine

Time of Search

$(D / M / Y)$

Databases of published research:

MEDLINE PubMed

$14 / 11 / 2017$

http://www.ncbi.nlm.nih.gov/pubmed/advanced

MEDLINE OvidSP

$17 / 11 / 2017$

http://ovidsp.tx.ovid.com/sp-

3.21.1b/ovidweb.cgi?\&S=CMAOFPFIOLDDCDGONCIKLDJCB

EOBAA $00 \& C=$ main\&tab $=$ search\&Main + Search + Page $=1$

Cochrane

Central Register

of Controlled

Trials

(CENTRAL)

LILACS (Latin

American and

Caribbean

Center on

Health Sciences

Google Scholar ttps://scholar.google.com.eg

OpenGrey

Clinical

trials.gov

ProQuest

Conference

Proceedings

Citation Index

The Cochrane Library

bases.bireme.br/cgi-

$\underline{\text { \&lang }=\mathrm{i}}$
$16 / 11 / 2017$

http://onlineliberary.wiley.com/cochraneliberary/search

$19 / 11 / 2017$

bin/wxislind.exe/iah/online/?IsisScript=iah/iah.xis\&base=LILACS www.opengrey.eu

www.clinicaltrials.gov

http://www.proquest.com/products-services/pqdtglobal.html

$18 / 11 / 2017$

http://thomsonreuters.com/en/products-services/scholarly-

$18 / 11 / 2017$

$20 / 11 / 2017$

$25 / 11 / 2017$

$18 / 11 / 2017$

$18 / 11 / 2017$

scientific-research/scholarly-search-and-discovery/conference-

proceedings-citation-index.html 
Additional hand search was done in some orthodontic journals including; American Journal of Orthodontics and Dentofacial Orthopedics, Angle Orthodontist, Seminars in Orthodontics, European journal of Orthodontics, Journal of Orthodontics and Journal of the World Federation of Orthodontists. References lists of related articles were hand searched as well to extract all the available studies related to our PIOS.The search strategy employed in the current systematic review utilized the keywords: (maxillary protrusion) (en mass* retract*) and (root resor*) as well as their synonyms (Figure 1).

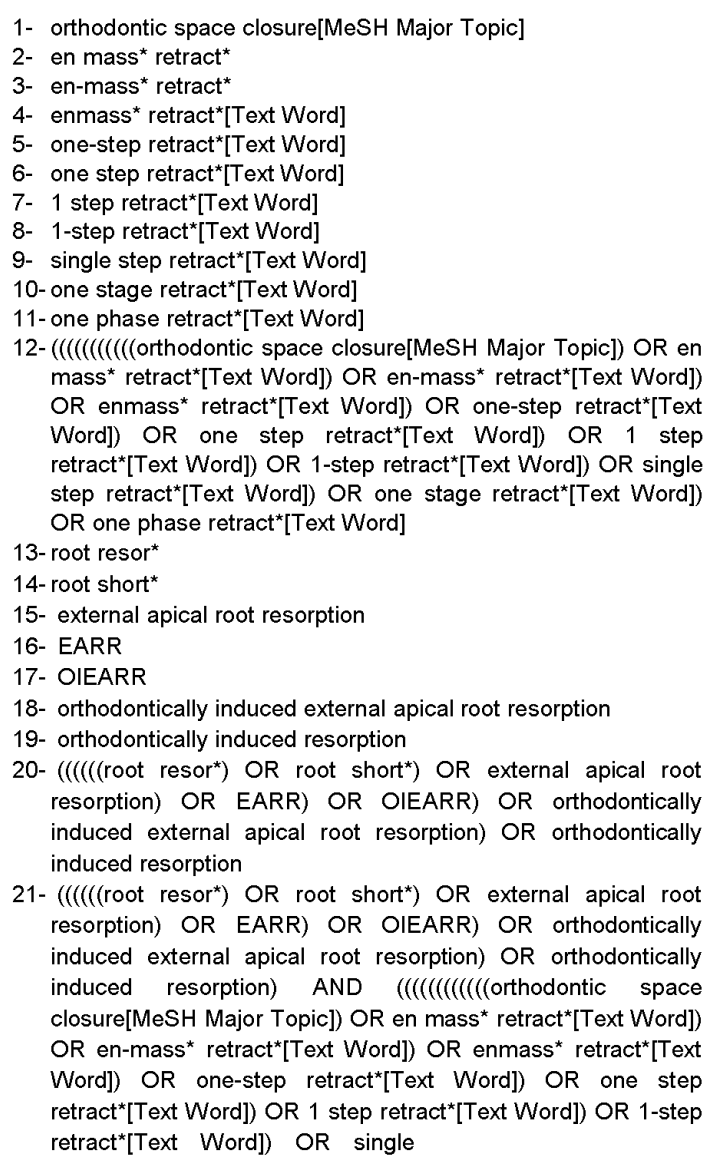

Figure 1: Search strategy for MEDLINE databases.

\begin{abstract}
step retract ${ }^{*}\left[\right.$ Text Word]) OR one stage retract* ${ }^{*}[$ Text Word]) OR one phase retract ${ }^{\star}[$ Text Word])

22- maxillary protrusion[Text Word]

23- maxillary proclination[Text Word]

24- maxillary procumbency[Text Word]

25- maxillary dentoalveolar protrusion[Text Word]

26- maxillary dental protrusion[Text Word]

27- maxillary alveolar protrusion[Text Word]

28- dental maxillary protrusion[Text Word]

29- $(((()((()(((($ maxillary protrusion[Text Word] $)$ OR maxillary proclination[Text Word]) OR maxillary procumbency[Text Word]) OR maxillary dentoalveolar protrusion[Text Word]) OR maxillary dental protrusion[Text Word]) OR maxillary alveolar protrusion[Text Word]) OR dental maxillary protrusion[Text Word])

30- $(((((()(((()(($ maxillary protrusion[Text Word] $)$ OR maxillary proclination[Text Word]) OR maxillary procumbency[Text Word]) OR maxillary dentoalveolar protrusion[Text Word]) OR maxillary dental protrusion[Text Word]) OR maxillary alveolar protrusion[Text Word]) OR dental maxillary protrusion[Text Word])) AND $(((()((()(($ orthodontic space closure[MeSH Major Topic]) OR en mass* retract*[Text Word]) OR en-mass* retract*[Text Word]) OR enmass* retract*[Text Word]) OR onestep retract*[Text Word]) OR one step retract*[Text Word]) OR 1 step retract ${ }^{\star}\left[\right.$ Text Word]) OR 1 -step retract ${ }^{\star}[$ Text Word]) OR single step retract ${ }^{\star}[$ Text $\left.W o r d]\right)$ OR one stage retract ${ }^{\star}[$ Text Word]) OR one phase retract ${ }^{\star}[$ Text Word])
\end{abstract}

Duplicates were removed after electronic and hand search, then studies were screened for inclusion by titles and abstracts. The eligibility criteria of the current research were designed to address the study design, participants, interventions, as well as the outcomes as shown in table 1 .

The exclusion criteriacomprised retrospective studies, clinical trials with insufficient sample size and unclear methodology, experimental as well as finite element model (FEM) studies, reviews, authors' replies as well as pilot studies and case reports. Exclusion criteria addressing the participants included growing subjects, or participants with previous history of trauma, endodontic treatment, impaction, habit or previous orthodontic treatment. Studies assessing en masse retraction with any additional surgical, pharmacological or physical interventions were excluded as well. 
The primary outcome measure was assessment of the incidence and amount of root resorption of maxillary and/ or mandibular teeth associated with en-masse retraction in adult patients with maxillary protrusion. Evaluating the amount of anterior teeth retraction and assessment of the pain associated with the retraction process were the secondary outcomes.

Data of the included studies were extracted using a customized data extraction sheet (table 3) developed by the same two authors separately and in case of disagreements, the third author was consulted. The methodological quality of the included studies was assessed using MINORS (Methodological Index For Non-Randomized Studies)and the quality of the studies were evaluated relative to the global ideal score (table 4).

\section{RESULTS:}

The current search protocol resulted in 1035 citations and ended with 1013 articles after duplicates' removal. Studies were evaluated primarily by their titles and irrelevant studies were excluded ending up with 125 articles. Following the eligibility criteria, studies were filtered on the basis of titles and abstracts where case reports/ series, reviews/ systematic reviews, books/E-books, experimental/FEM and studies with other languages were excluded.

Full text reading of the 21 articles that resulted from the previous step was done. The eligibility criteria were assessed among these studies where 9 studies were retrospective (16-24), 2 study had unclear study design and methodology $(25,26), 3$ studies employed growing subjects $(12,14,27), 4$ studies didn't assess any of the primary or secondary outcomes concerned and so were excluded (13, 28-30). Additionally, 1 study was found to be a preliminary study and was excluded as well (31). Thus, 19 studies were excluded after their assessment for eligibility and only 2 studies were included in the qualitative synthesis $(32,33)$. The article selection process is illustrated in the PRISMA flow diagram (Figure 2).

The 2 included studies were a non-randomized clinical study (32) and a clinical trial (33). Data from both studies were pooled into a customized data extraction sheet (Table 3). The sheets categorized the characteristics of the included studies as follows: Aim of study, Study design/settings, Participants' criteria and Demographic findings, Detailed interventions' criteria and Methodologic findings, Primary outcome, Secondary outcomes and Authors' conclusions.

The MINORS tool was used by both investigators independently to evaluate and score the quality of both studies through twelve criteria and the resultant scores were 17 for the first study (32)and 14 for the second (33)(Table 4). The ideal global score of the MINORS tool is 24 for comparative studies and 16 for the non-comparative studies (34). The 2 studies included in the current review were less than that score denoting the fair quality of the findings concluded.

Furthermore, the studies included were heterogeneous and the outcomes assessed were incomparable as one study measure the root resorption associated with en-masse retraction for the maxillary and mandibular incisors (32) while the other study evaluated the root shortening of canines, premolars and molars for both arches (33). Thus, a meta-analysis of the included studies was not possible. 
Table 3: Characteristics of included studies

Study

Aim of the study

Study design/ settings

Participants:

1- Characterist ics

2- Angle's classificatio $n$ of malocclusio $n$

3- Sample size

4- Number of participants/ group

5- Mean age of participants (years)/grou $p$ at $T 1$ (before treatment)

6- Gender of participants/ group

Intervention:

1- Intervention
Huang et el. 2010

Kalha et al. 2010

To investigate the comparative amount of root shortening between two-step and en masse space closure procedures.

Non RCT/ not mentioned.

- No evidence of resorption on the pretreatment panoramic radiographs

- No contributing history of trauma;

- No severely dilacerated incisor roots, anodontia, or impacted canines;

- Complete root formation of incisors at the start of treatment;

- Intact and caries-free incisors;

- No endodontically treated incisors;

- Requiring extraction of all four first premolars; and space closure with moderate anchorage.

- Angle Class I or II malocclusion

- 16 class I \& 10 class II in G1

- 19 class I \& 7 class II in G2

- 52 patients

- 26 in intervention group $(\mathrm{G} 1) / 26$ in control group $(\mathrm{G} 2)$

- $\quad 15.6 \pm 1.9$

- $\mathrm{G} 1=15.8 \pm 1.8$

- $\mathrm{G} 2=15.4 \pm 1.9$

- Not mentioned

- 20 patients

- 10 patients were evaluated for rate of tooth movement, anchorage loss and root resorption using Hycon screw for anterior space closure.

- 10 patients were employed for assessing the ALP activity where 5 patients had anterior space closure with Hycon screw \& 5 patients with active tie-backs.

- $\quad 19.9 \pm 3.8$

- For the $2^{\text {nd }}$ part of the study (ALP activity) mean age was $20.6 \pm 3.2$

- 18 females \& 8 males in G1

- 18 females \& 8 males in $\mathrm{G} 2$

10 males \& 10 females.

- In the $2^{\text {nd }}$ part of the study (ALP activity) (6 females and 4 males)

- Group 1 (G1): two-step retraction
- For the first part of the study all 
in each

group.

\section{2- Type of} brackets

3- Size of main archwire during retraction

4- Means of retraction:

a) Point of applicatio $n$ on main archwire

b) Retraction tool

c) Force of retraction

d) Force activation/ recalibrati on

5- Anchorage means:

6- Timing of taking records

7- Teeth assessed for root resorption

8- How was root resorption assessed
- Group 2 (G2): en-masse retraction

- 0.022-inch bracket

slot multibondedpreadjusted appliances (OPA-K; Tomy, Ohkuma-machi, Japan)

- Rectangular stainless steel $\operatorname{archwire}(\mathbf{0 . 0 1 8} " \times \mathbf{0 . 0 2 5} ")$

a) G1:NiTi coil spring ligated with a wire to the canine bracket hook or archwire hook soldered mesial to the canine/ G2: $\mathrm{Ni}-\mathrm{Ti}$ coil springs were placed across the extraction sites from the buccal tube hook on the first molar to the archwire hook, soldered mesially to the canine.

b) 0.012-inch nickel titanium (Ni-Ti) coil springs (Grikin, Beijing, China)

c) $150 \mathrm{gm}$ in both groups

d) Springs were checked to confirm 150-g activation every 4 weeks.

- Moderate anchorage (Details not mentioned).

- Records were taken before \& after space closure (before the finishing procedure)

- Central \& lateral incisors

- Maxillary \& mandibular

- Panoramic radiographs were taken with the same radiographic machine (Orthophos; Sirona, Bensheim, Germany) and a standardized technique.

- Tooth length was measured from the apex to the incisal edge along the longitudinal axis of each tooth as accurately as possible. All participants had space closure using Hycon screw.

- $\quad$ For the second part of the study (ALP activity):5 participants had space closure using Hycon screw and the rest used active tie-backs.

- 0.022-inch slot brackets. (Type and company was not mentioned)

- Stainless steel archwires(0.021" $\times$ 0.025)

a) Loop mesial to canines.

b) Hycon screw

c) 205 gm per activation

d) Patients were advised to activate the screw half a turn every 3 days until space closure was complete.

- Banding all second molars and placing TPA \& lingual arch.

- After levelling \& alignment stage and after treatment.

- Canine \& molar/premolar region

- Maxillary \& mandibular

- RVGs were taken with an UltraCam (Ultrak Inc.) \& projected on a computer screen using Dexis 3.0 software.

- The canine \& molar/premolar regions were scored according to:

$0=$ No apical root resorption

$1=$ Slight blunting of the apex

$2=$ Moderate blunting of the apex up to 
measurements were performed by the one fourth of the root length

same clinician with the software (Sidexis; Sirona, Bensheim, Germany) with an accuracy of 0.01 $\mathrm{mm}$.

- Before panoramic radiographs were obtained, eight metal rods were ligated to the incisors' brackets with ligature wires. The metal rods, which were made of 0.018-inch stainless steel wires, were $8.0 \mathrm{~mm}$ or $6.0 \mathrm{~mm}$ in length, as measured with vernier caliper, and were ligated to maxillary and mandibular incisor brackets, respectively.

Outcomes:

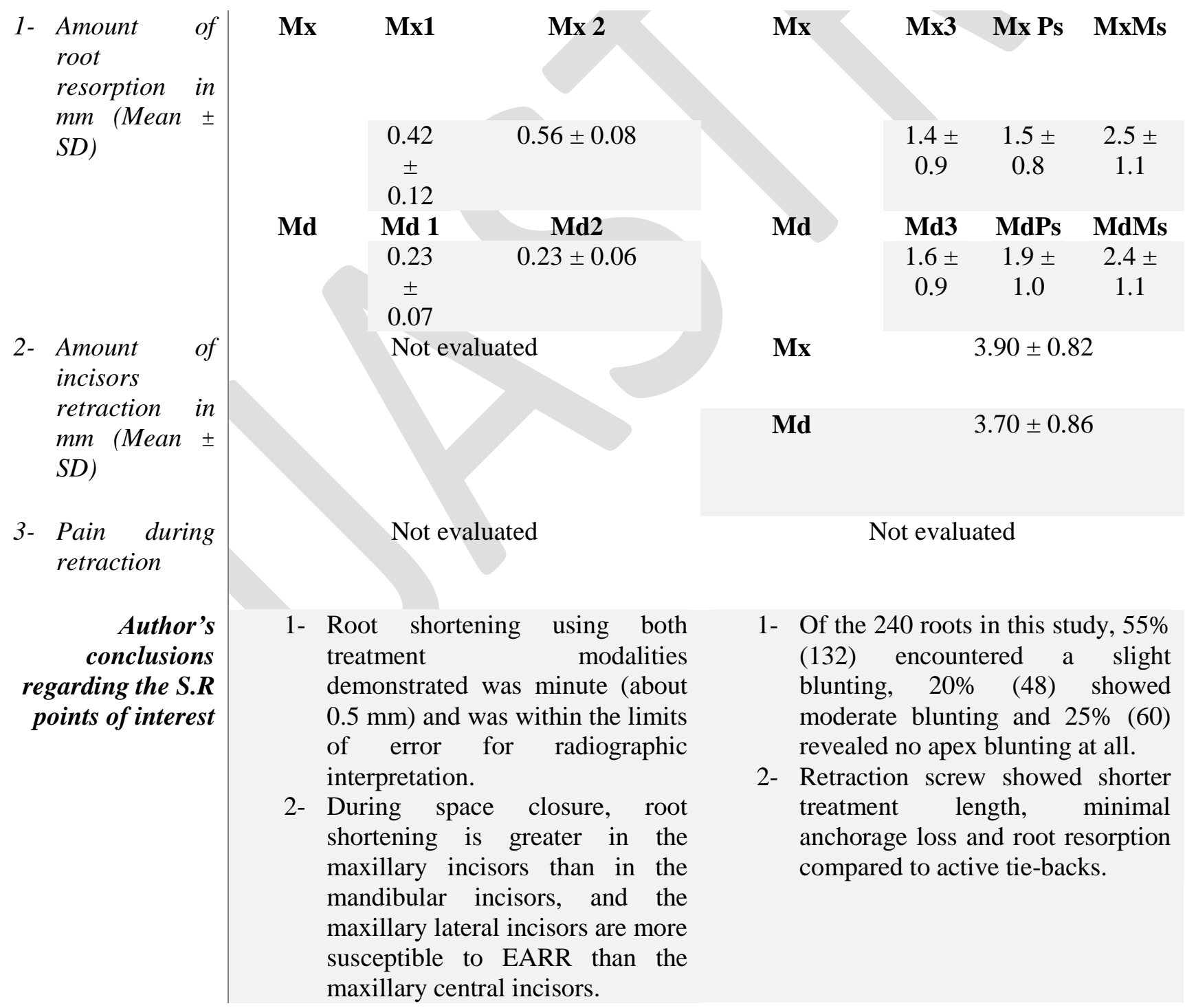

3 = Excessive blunting of the apex beyond one fourth of the root length

- The resorption scores were added for all the teeth examined, the total resorption score for each patient was determined

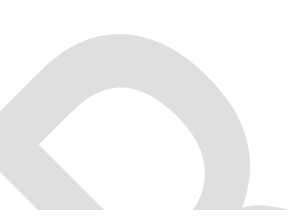

Md3 MdPs MdMs

$1.6 \pm \quad 1.9 \pm 2.4 \pm$

$3.90 \pm 0.82$

. 
Table 4: MINORS of the included studies

MethodologicalMethods

(Huang et al.

2010)

Score

\begin{tabular}{l|lcc}
1 & A clearly stated aim: & 2 & 2 \\
2 & Inclusion of consecutive patients: & 2 & 2 \\
3 & Prospective collection of data: & 2 & 2 \\
4 & $\begin{array}{l}\text { Endpoints appropriate to the aim of } \\
\text { the study: }\end{array}$ & 2 & 2 \\
5 & Unbiased assessment of the study & 0 & 0 \\
& endpoint: & 2 & 2 \\
6 & Follow up period appropriate to the & & \\
& aim of the study: & 0 & 0 \\
7 & Loss of follow up less than 5 \%: & 0 & 0 \\
8 & Prospective calculation of the study & & 0 \\
& size: & 2 & 2 \\
9 & An adequate control group: & 2 & 0 \\
10 & Contemporary groups: & 1 & 2 \\
11 & Baseline equivalence of groups: & 2 & 14 \\
12 & Adequate statistical analysis: & 17 &
\end{tabular}



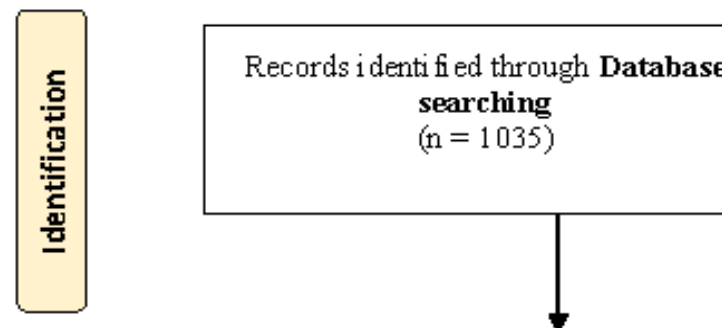

$(\mathrm{n}=1035)$
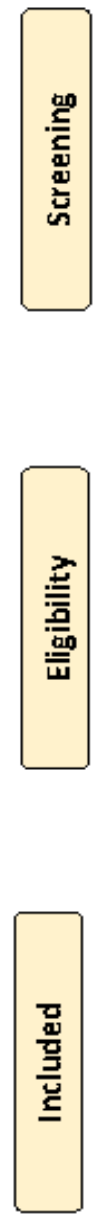

Records exchuded not relevant

to the subject

$(\mathrm{n}=885)$

Full-text articles excluded, case reports/ series, revi ews/

Full-text articles assessed for eligibility

$(n=128)$

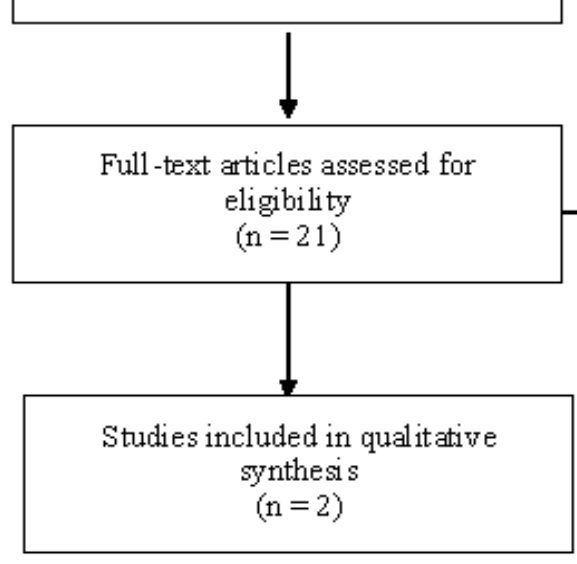
systematic reviews, books/Ebooks, language restriction, experimental/ $\mathrm{FEM}$ $(n=107)$

Full-text articles excluded with reasons:

Retrospective studies ( $n=9$ )

Growing subjects $(n=3)$

Unclear study design/methodology $(\mathrm{n}=2)$ Outcomes not assessed ( $\mathrm{n}=$ 4)

Filot/Preliminary study $(\mathrm{n}=$ 1)

$$
(n=19)
$$

Figure 2: PRISMA Flow Diagram 


\section{The amount of root resorption:}

Despite root resorption associated with en-masse retraction was evaluated in both studies included in the qualitative synthesis, different teeth were evaluated. The first study (32)comprised 52 participants and root resorption of maxillary and mandibular incisors was evaluated showing an average root shortening of $0.42 \pm 0.12 \mathrm{~mm}$ and $0.23 \pm 0.07 \mathrm{~mm}$ for maxillary and mandibular central incisors. Maxillary lateral incisors showed $0.56 \pm 0.08 \mathrm{~mm}$ root resorption while the mandibular showed $0.23 \pm$ $0.06 \mathrm{~mm}$.

Canines, premolars and molars were assessed for root resorption in the second study(33) which employed 20 participants. The upper and lower canines exhibited $1.4 \pm 0.9 \mathrm{~mm}$ and $1.6 \pm 0.9 \mathrm{~mm}$. meanwhile, upper premolars showed $1.5 \pm 0.8 \mathrm{~mm}$ and lower premolars revealed resorption of $1.9 \pm 1.0$ $\mathrm{mm}$ of their roots. The molars revealed the greatest amount of root shortening where $2.5 \pm 1.1 \mathrm{~mm}$ and 2.4 $\pm 1.1 \mathrm{~mm}$ were observed in the maxillary and mandibular molars respectively.

\section{The amount of anterior teeth retraction:}

This finding was only assessed in one study (33) denoting an overall average retraction of maxillary anterior teeth using en-masse retraction technique with the Hycon screw device of $3.90 \pm 0.82$ $\mathrm{mm}$ and lower anterior teeth were retracted by an average of $3.70 \pm 0.86 \mathrm{~mm}$.

The final outcome to be assessed by the current review is the level of pain perceived using enmasse retraction technique and its pattern of severity. However, none of the included studies assessed this finding.

\section{DISCUSSION:}

The present systematic review was performed to systematically analyze the incidence and the degree of external apical root resorption with en-masse retraction in patients having maxillary protrusion. Although orthodontic treatment may predispose to root resorption specially with long treatment duration and increased teeth movement $(16,35-37)$,limited data were reported in previous reviews regarding the effect of orthodontic interventions on root resorption and its management $(38,39)$ and no previous studies systematically reviewed its correlation with en-masse retraction techniques.

For the current review, not only randomized clinical trials (RCTs) and quasi randomized clinical trials (q RCTs) were included but also non-randomized clinical trials (non-RCTs), prospective controlled clinical trials (CCTs) and clinical studies of sufficient sample size and suitable methodology were included. Despite the high quality of outcomes reported from RCTs and the recommendations of the Cochrane collaboration to include only RCTs to ensure robust and solid conclusions from systematic reviews(40), another opinion suggested that q RCTs and non-RCTs should only be included if there are only few or no RCTs which was the case in this review. According to Liberatiet al. 2009 the inclusion of non-randomized studies is recommended in some systematic reviews that evaluate the effects of health care interventions(41).

The eligibility criteria of the studies were set so that only studies having non-growing participants were included to eliminate the effect of age on the tissue response as a confounding factor $(42,43)$. Additionally, all factors predisposing to root shortening were set in the exclusion criteria including previous incidence of trauma, previous impaction and root shortening as well as preceding orthodontic treatment or systemic allergic condition (43).

Regarding the intervention, the standard and common management of patients having maxillary protrusion is extraction of first premolars and retraction of anterior teeth. Thus, this was the intervention protocol selected for including studies in the current review. Metal brackets using either ROTH or MBT 
prescription were included but it was planned to use sub-group analysis to separate the findings resulting from using both bracket systems. Studies managing maxillary protrusion cases with another extraction protocol or using lingual, ceramic, bi-dimensional brackets were excluded to rule out any external factor that might affect the incidence and severity of root resorption, the amount of anterior teeth retraction as well as the pain felt by patients.

Recently, many studies have been interested in modalities that could accelerate tooth movement and reduce adverse effects including surgical, physical, and pharmacological interventions (44). None of the studies using any of these interventions were retrieved in our review as the effectiveness and possible effects of such techniques is still under investigation and the scope of this review was to investigate the effect of regular en-masse retraction techniques.

Following the stated criteria only 2 studies were eligible for qualitative analysis(32, 33). It is worth mentioning that the study conducted by Guoet al. 2011seemed to fit the inclusion criteria however upon full text article retrieval many study details were unclear including the bracket slot size used, the main archwire used for retraction, the timing of force activation and the size of the miniscrews used as anchorage means. Moreover, it was stated that the study used "retrospective 3D registration" (25). We failed to get a clue regarding the points of interest after trying to contact the author via e-mail.

Similarly, Luniet al. 2017performed a recent study that was primarily included in the current review, however, some fundamental details of the study were unclear including the participants' gender, the technique and archwire used for retraction as well as the study design ${ }^{(26)}$. Hence, these studies were excluded from our qualitative synthesis. Four of the studies primarily included on the basis of title and abstracts $(13,28-30)$ were later excluded because neither the primary outcome nor any of the secondary outcomes were assessed in these studies.

Quality assessment of the included studies was performed using MINORS scoring tool. This tool proved to be excellent for assessing non-randomized interventional studies (45). Twelve criteria were followed to evaluate the methodological quality of both studies where the first eight criteria were used to evaluate the comparative as well as non-comparative studies while the last four criteria were set only to assess the comparative studies. Following the guidelines set by the MINORS tool (34), both included studies' scores were below the ideal global score with the study conducted by Huang et al. 2010 showed a relatively superior study quality.

Root resorption associated with en-masse retraction was reported in both studies, yet findings of both studies could not be combined nor applied similarly. The inherent reason is that different main archwires were utilized during en-masse retraction which could have affected the resulting root resorption due to different torque expression. Huang et al. 2010 used $(0.018$ " $\times 0.025$ ") main archwire within 0.022 " slot brackets, while Kalhaet al. 2010 used $(0.021 " \times 0.025)$ within the same slot size. Conclusively, more root resorption was found in the second study although different teeth were evaluated.

Adding to that, different force magnitudes and tools of retraction were employed with $150 \mathrm{gm}$ force applied using NiTi coil springs in the first study (32), while an average of $205 \mathrm{gm}$ retraction force utilizing Hycon screw was used in the second study (33) with different timings of activation. The first study assessed root resorption on incisors while the second evaluated the same finding on canines, premolars and molars. Huang $\boldsymbol{e t}$ al. 2010 reported more root resorption for maxillary than mandibular incisors, with the lateral incisor scoring the greatest amount of root resorption. However, all reported measurements were clinically insignificant as they were all less than $0.5 \mathrm{~mm}$ (32).

Meanwhile, Kalhaet al. 2010 revealed that the greatest root resorption was scored for maxillary first molars of more than $2.5 \mathrm{~mm}$. Significant root shortening was reported by this study where the least amount recorded was around $1.4 \mathrm{~mm}$ and this could be attributed to the reasons mentioned beforehand(33). 
The secondary outcomes were not assessed in the first study (32), while amount of anterior teeth retraction was reported by the second study of around $3.9 \mathrm{~mm}$ using the Hycon screw device with TPA, Lingual arch and banding second molars as a means of anchorage(33).

\section{CONCLUSIONS:}

1- No high quality evidence was found regarding the root resorption associated with en-masse retraction in adults having maxillary protrusion.

2- From the available evidence, no clinically significant resorption of the maxillary and mandibular incisors was associated with en-masse retraction using $150 \mathrm{gm}$ retraction force, and 0.018 " $\times 0.025$ " main archwire within 0.022 " bracket slot. Relatively increased root resorption was found in maxillary lateral incisors recording a mean of $0.56 \pm 0.08 \mathrm{~mm}$.

3- Evidence of fair quality suggested that increased amounts of root resorption were scored for the maxillary molars when compared to canines, premolars of both arches and mandibular first molars scoring an average of $2.5 \pm 1.1 \mathrm{~mm}$.

4- Increased amounts of root resorption were associated with using heavy archwires $\left(0.021^{\prime \prime} \times 0.025\right)$ and/ or heavier forces.

5- The findings of this systematic review should be interpreted with caution due to the limited quality of the included studies and inherent heterogeneity. Properly designed randomized controlled trials must be conducted to reach a robust and sound conclusion.

\section{REFERENCES:}

[1] Brezniak N and Wasserstein A. Root resorption after orthodontic treatment: Part 2. Literature review. American Journal of Orthodontics and Dentofacial Orthopedics. 1993;103(2):138-46.

[2] Harris EF, Kineret SE and Tolley EA. A heritable component for external apical root resorption in patients treated orthodontically. American journal of orthodontics and dentofacial orthopedics. 1997;111(3):301-9.

[3] Owman-Moll P, Kurol J and Lundgren D. Continuous versus interrupted continuous orthodontic force related to early tooth movement and root resorption. The Angle orthodontist. 1995;65(6):395-401; discussion -2 .

[4] Kurol J, Owman-Moll P and Lundgren D. Time-related root resorption after application of a controlled continuous orthodontic force. American journal of orthodontics and dentofacial orthopedics. 1996;110(3):303-10.

[5] Sameshima GT and Sinclair PM. Predicting and preventing root resorption: Part I. Diagnostic factors. American journal of orthodontics and dentofacial orthopedics. 2001;119(5):505-10.

[6] Mirabella AD and Artun J. Prevalence and severity of apical root resorption of maxillary anterior teeth in adult orthodontic patients. European journal of orthodontics. 1995;17(2):93-9.

[7] Parker RJ and Harris EF. Directions of orthodontic tooth movements associated with external apical root resorption of the maxillary central incisor. American journal of orthodontics and dentofacial orthopedics. 1998;114(6):677-83.

[8] Harry MR and Sims MR. Root resorption in bicuspid intrusion. A scanning electron microscope study. The Angle orthodontist. 1982;52(3):235-58.

[9] Chan E and Darendeliler MA. Physical properties of root cementum: Part 5. Volumetric analysis of root resorption craters after application of light and heavy orthodontic forces. American journal of orthodontics and dentofacial orthopedics. 2005;127(2):186-95.

[10] Linge BO and Linge L. Apical root resorption in upper anterior teeth. European journal of orthodontics. 1983;5(3):173-83. 
[11] Ramanathan C and Hofman Z. Root resorption during orthodontic tooth movements. European journal of orthodontics. 2009;31(6):578-83.

[12] Xu TM, Zhang X, Oh HS, Boyd RL, Korn EL, and Baumrind S. Randomized clinical trial comparing control of maxillary anchorage with 2 retraction techniques. American journal of orthodontics and dentofacial orthopedics. 2010;138(5):544.e1-9; discussion -5.

[13] Al-Sibaie S and Hajeer MY. Assessment of changes following en-masse retraction with miniimplants anchorage compared to two-step retraction with conventional anchorage in patients with class II division 1 malocclusion: a randomized controlled trial. European journal of orthodontics. 2014;36(3):275-83.

[14] Ajmera S, Shivanand V, and Ganeshkar SV. Volumetric evaluation of root resorption during orthodontic treatment. Journal of clinical orthodontics. 2014;48(2):113-9.

[15] Antoszewska-Smith J, Sarul M, Lyczek J, Konopka T, and Kawala B. Effectiveness of orthodontic miniscrew implants in anchorage reinforcement during en-masse retraction: A systematic review and meta-analysis. American journal of orthodontics and dentofacial orthopedics. 2017;151(3):440-55.

[16] DeShields RW. A study of root resorption in treated Class II, Division I malocclusions. The Angle orthodontist. 1969;39(4):231-45.

[17] Janson GR, De Luca Canto G, Martins DR, Henriques JF, and De Freitas MR. A radiographic comparison of apical root resorption after orthodontic treatment with 3 different fixed appliance techniques. American journal of orthodontics and dentofacial orthopedics. 2000;118(3):262-73.

[18] Yao CC, Lai EH, Chang JZ, Chen I, and Chen YJ. Comparison of treatment outcomes between skeletal anchorage and extraoral anchorage in adults with maxillary dentoalveolar protrusion. American journal of orthodontics and dentofacial orthopedics. 2008;134(5):615-24.

[19] Liou EJand Chang PM. Apical root resorption in orthodontic patients with en-masse maxillary anterior retraction and intrusion with miniscrews. American journal of orthodontics and dentofacial orthopedics. 2010;137(2):207-12.

[20] Lynch JR. DMD, "Three-Dimensional Evaluation of the Effect of Maxillary Incisor Retraction on the Palatal Bone and Root Resorption" [Master's Thesis]: University of Connecticut Graduate School; 2011.

[21] Ahn HW. Evaluation of the Alveolar Bone and Roots of the Maxillary Anterior Teeth before and after En-Masse Retraction Using Cone-Beam Computed Tomography [Master's thesis]: Seoul National University; 2012.

[22] Ahn HW, Moon SC, and Baek SH. Morphometric evaluation of changes in the alveolar bone and roots of the maxillary anterior teeth before and after en masse retraction using cone-beam computed tomography. The Angle orthodontist. 2013;83(2):212-21.

[23] Monga N, Kharbanda OP, and Samrit V. Quantitative and qualitative assessment of anchorage loss during en-masse retraction with indirectly loaded miniscrews in patients with bimaxillary protrusion. American journal of orthodontics and dentofacial orthopedics. 2016;150(2):274-82.

[24] Barros SE, Janson G, Chiqueto K, Baldo VO, and Baldo TO. Root resorption of maxillary incisors retracted with and without skeletal anchorage. American journal of orthodontics and dentofacial orthopedics. 2017;151(2):397-406.

[25] Guo QY, Zhang SJ, Liu H, Wang CL, Wei FL, and Lv T. Three-dimensional evaluation of upper anterior alveolar bone dehiscence after incisor retraction and intrusion in adult patients with bimaxillary protrusion malocclusion. Journal of Zhejiang University Science B. 2011;12(12):990-7.

[26] Luni U, Mahmood NE, Ehsan AA, Sakrani H. Apical root resorption in Class II Div 1 malocclusion patients during en-masse maxillary anterior retraction with mini-screws2017. 
[27] Castro IO, Alencar AH, Valladares-Neto J, and Estrela C. Apical root resorption due to orthodontic treatment detected by cone beam computed tomography. The Angle orthodontist. 2013;83(2):196-203.

[28] Upadhyay M, Yadav S, Nagaraj K, and Patil S. Treatment effects of mini-implants for en-masse retraction of anterior teeth in bialveolar dental protrusion patients: a randomized controlled trial. American journal of orthodontics and dentofacial orthopedics. 2008;134(1):18-29.e1.

[29] Upadhyay M, Yadav S, and Patil S. Mini-implant anchorage for en-masse retraction of maxillary anterior teeth: a clinical cephalometric study. American journal of orthodontics and dentofacial orthopedics. 2008;134(6):803-10.

[30] Basha AG, Shantaraj R, and Mogegowda SB. Comparative study between conventional en-masse retraction (sliding mechanics) and en-masse retraction using orthodontic micro implant. Implant dentistry. 2010;19(2):128-36.

[31] Oliveira TMF, Claudino LV, Mattos CT, and Sant'Anna EF. Maxillary dentoalveolar assessment following retraction of maxillary incisors: a preliminary study. Dental press journal of orthodontics. 2016;21:82-9.

[32] Huang Y, Wang XX, Zhang J, and Liu C. Root shortening in patients treated with two-step and en masse space closure procedures with sliding mechanics. The Angle orthodontist. 2010;80(3):492-7.

[33] Kalha AS, Kachiwala VA, Govardhan SN, McLaughlin RP, and Khurshaid SZ. Redefining orthodontic space closure: sequential repetitive loading of the periodontal ligament--a clinical study. World journal of orthodontics. 2010;11(3):221-9.

[34] Slim K, Nini E, Forestier D, Kwiatkowski F, Panis Y, and Chipponi J. Methodological index for non-randomized studies (minors): development and validation of a new instrument. ANZ journal of surgery. 2003;73(9):712-6.

[35] Reitan K. Initial tissue behavior during apical root resorption. The Angle orthodontist. 1974;44(1):68-82.

[36] Goldson L, and Henrikson CO. Root resorption during Begg treatment: A longitudinal roentgenologic study. American Journal of Orthodontics. 1975;68(1):55-66.

[37] Lopatiene K, and Dumbravaite A. Risk factors of root resorption after orthodontic treatment. Stomatologija. 2008;10(3):89-95.

[38] Jayaratne YSN, Uribe F, and Janakiraman N. the effect of mini-implant supported anchorage on maxilary incisor position during retraction-A systematic review.Journal of Istanbul University Faculty of Dentistry. 2017;51(3 Suppl 1).

[39] Ahangari Z, Nasser M, Mahdian M, Fedorowicz Z, and Marchesan MA. Interventions for the management of external root resorption. Cochrane Database of Systematic Reviews. 2015(11).

[40] Higgins JPT GS. Cochrane Handbook for Systematic Reviews of Interventions Version 5.1.0 [updated March 2011]. The Cochrane Collaboration. 2011.

[41] Liberati A AD, Tetzlaff J, Mulrow C, Gøtzsche PC, Ioannidis JPA, Clarke M, Devereaux PJ, Kleijnen J, and Moher D. . The PRISMA Statement for Reporting Systematic Reviews and MetaAnalyses of Studies That Evaluate Health Care Interventions: Explanation and Elaboration. PLoS Med 2009; 6(7)(e1000100.).

[42] Ren Y, Maltha JC, Liem RS, Stokroos I, and Kuijpers-Jagtman AM. Age-dependent external root resorption during tooth movement in rats. Acta odontologica Scandinavica. 2008;66(2):93-8.

[43] Topkara A, Karaman AI, and Kau CH. Apical root resorption caused by orthodontic forces: A brief review and a long-term observation. European journal of dentistry. 2012;6(4):445-53.

[44] Long H, Pyakurel U, Wang Y, Liao L, Zhou Y, and Lai W. Interventions for accelerating orthodontic tooth movement: a systematic review. The Angle orthodontist. 2013;83(1):164-71. 
[45] Zeng X, Zhang Y, Kwong JS, Zhang C, Li S, and Sun F. The methodological quality assessment tools for preclinical and clinical studies, systematic review and meta-analysis, and clinical practice guideline: a systematic review. Journal of evidence-based medicine. 2015;8(1):2-10. 